\title{
Plasma amino acid levels in a cohort of patients in Turkey with classical phenylketonuria
}

\author{
Kazanasmaz Halil1,*, Karaca Meryem²
}

\begin{abstract}
Background: In patients with phenylketonuria, the central nervous system is adversely affected by noncompliance with diet. The levels of phenylalanine and many different amino acids (AAs) in the plasma of patients with phenylketonuria can be measured simultaneously.

Objectives: To measure the blood plasma levels of neurotransmitter AAs in a cohort of patients in Sanliurfa province, Turkey, with phenylketonuria for use as a support parameter for the follow-up of patients.

Methods: The phenylketonurics that we followed $(\mathrm{n}=100)$ were divided into 2 groups according to their compliance with their dietary treatment. Plasma AA analysis results of phenylketonurics were compared with those of healthy children in a control group $(\mathrm{n}=50)$.

Results: In the diet incompliant group $(\mathrm{n}=56)$, the mean levels of $\gamma$-aminobutyric acid (GABA; $0.96 \pm 1.07 \mu \mathrm{mol} / \mathrm{L})$ and glycine $(305.1 \pm 105.19 \mu \mathrm{mol} / \mathrm{L})$ were significantly higher than those in the diet compliant group $(\mathrm{n}=44$; GABA $P=0.005$, glycine $P<0.001$ ) and in the control group (GABA and glycine $P<0.001$ ), whereas the mean levels of glutamic acid $(39.01 \pm 22.94 \mu \mathrm{mol} / \mathrm{L})$ and asparagine $(39.3 \pm 16.89 \mu \mathrm{mol} / \mathrm{L})$ were lower $(P<0.001)$ in the diet incompliant group. A positive correlation was observed between the levels of phenylalanine and GABA and glycine. A negative relationship was found between the levels of phenylalanine and glutamic acid and asparagine.

Conclusions: A relationship exists between the levels of plasma phenylalanine in a cohort of phenylketonurics in Sanliurfa province, Turkey, and the levels of some excitatory and inhibitory AAs. Excitatory and inhibitory AA levels in plasma may be used as support parameters in the follow-up of patients with phenylketonuria.
\end{abstract}

Keywords: amino acids; chromatography; diet therapy; neurotransmitter agents; phenylketonuria

Phenylketonuria is an inherited autosomal recessive disease of phenylalanine (Phe) metabolism resulting from variants in genes-encoding phenylalanine hydroxylase. Phenylalanine hydroxylase transforms Phe into tyrosine (Tyr) on the condition that cofactors tetrahydrobiopterin, molecular oxygen, and iron are presented in the environment [1]. Disorders of Phe metabolism cause a significant increase in the concentration of Phe in the blood and brain tissue, which may lead to various neurocognitive and neuromotor disorders, if not treated in the neonatal period [2-5]. Dietary treatment is administered to prevent the development of the adverse effects of phenylketonuria, which is extremely important. Currently, the dietary treatment is supplemented with other amino acids (AAs) in conjunction with the restricted Phe intake to support growth and to maintain a healthy body composition that forms the basis of treatment $[2,6]$. In patients with phenylketonuria

*Correspondence to: Kazanasmaz Halil, Department of Pediatrics, Faculty of Medicine, Harran University, Osmanbey Campus, Sanliurfa 63000, Turkey, e-mail: kazanasmazhalil@harran.edu.tr 'Department of Pediatrics, Faculty of Medicine, Harran University, Sanliurfa 63300, Turkey ${ }^{2}$ Department of Pediatric Metabolism Disorders, Faculty of Medicine, Harran University, Sanliurfa 63300, Turkey

D Open Access. ๑ 2020 Halil and Meryem, published by Sciendo. (cc) Br-Nc-ND This work is licensed under the Creative Commons Attribution NonCommercial-NoDerivatives 4.0 License. 
who receive the dietary treatment, the plasma Phe levels of $120-360 \mu \mathrm{mol} / \mathrm{L}$ are targeted for children aged $<12$ years, while the plasma Phe levels of $120-600 \mu \mathrm{mol} / \mathrm{L}$ are targeted for children aged $\geq 12$ years $[2,7,8]$.

An accurate analysis of the types and amounts of AAs is extremely important in determining the efficacy of dietary treatment administered to phenylketonurics. Liquid chromatography-mass spectrometry (LC-MS) is an analytical technique widely used for AA analysis. Recently, LC-MS has been further developed, and a method comprising a combination of two mass analyzers in one mass spectrometry instrument (LC-MS/MS) has been used [9-11].

AAs and their derivatives may play a role in the pathophysiology of neuropsychiatric disorders through variations in molecular mechanisms [12,13]. Through LC-MS/MS analysis, the plasma levels of various AAs, such as $\gamma$-aminobutyric acid (GABA), glycine (Gly), glutamic acid (Glu), and asparagine (Asn), which play important roles in the central nervous system (CNS), can be easily measured. In phenylketonurics, various neurological problems may develop with the increased Phe level in the blood and brain tissue $[14,15]$.

We sought to investigate the effects of the increased plasma level of Phe on the levels of other AAs in the plasma (especially excitatory and inhibitory AAs) and whether the levels of other AAs can be used as support parameters for follow-up of patients.

\section{Methods}

After approval of the study protocol by the Harran University Ethics Committee for Human Research (certificate of approval No. E40428; approval date October 4, 2018, session 10/40428) following the Declaration of Helsinki and its contemporary amendments, and after obtaining written informed consent to participate in the study from the parents of all patients and controls included, we conducted a study in a community in Sanliurfa province, Turkey. All participants with phenylketonuria included in the study were followed up regularly from October 15, 2017 to October 15, 2018 in the pediatric metabolic diseases clinic. Data were obtained retrospectively from each phenylketonuria patient's medical record at the time of their most recent visit, which included age, sex, height, weight, adherence to dietary treatment, and plasma AA analysis results. Blood samples were collected from age-matched healthy control patient-participants attending the Pediatric Metabolism Disorders clinic but with normal results and volunteered by their parents in the month from 15 October 2018 to 15 November 2018, and data were obtained, which included age, sex, height, weight, and plasma AA analysis results on the same day. The phenylketonurics were divided into 2 groups according to their adherence to dietary treatment: those who adhered [diet compliant (DC)] and who had not adhered to their diet [diet incompliant (DIC)], and plasma AA analysis results were compared with those of healthy children in the control group. The participants included in the control group were selected from among healthy children who did not have any disease or pathological condition. The patients in the DC group were selected among the patients who had the target plasma levels of Phe $(<12$ years $120-360 \mu \mathrm{mol} / \mathrm{L}, \geq 12$ years $120-600 \mu \mathrm{mol} / \mathrm{L}$ ) in all 3 consecutive follow-up examinations within the last 6 months. The patients in the DIC group were selected among the patients who exceeded the target plasma levels of Phe $(<12$ years $>360 \mu \mathrm{mol} / \mathrm{L}, \geq 12$ years $>600 \mu \mathrm{mol} / \mathrm{L})$ as determined in all 3 consecutive follow-up examinations within the last 6 months. Patients who did not attend the followup examinations ( 3 times in the last 6 months) were excluded from the study. The plasma samples taken for the LC-MS/MS analysis were analyzed immediately after they are collected. Informed consent from the parents of all participants included in the study was obtained and documented before the venous blood samples were taken.

\section{LC-MS/MS plasma AA analysis sample collection and the study protocol}

For plasma AA analysis, we used a Quantitative Amino Acids Analysis kit (Jasem, Istanbul, Turkey). Venous blood samples $(2 \mathrm{~mL})$ were collected into heparinized tubes and centrifuged. After separating $50 \mu \mathrm{L}$ of the centrifuged plasma and placing it into a tube (Eppendorf, Hamburg, Germany), $50 \mu \mathrm{L}$ of AA internal standard (ISTD) was added to it and the mixture was vortexed for $5 \mathrm{~s}$. Finally, $700 \mu \mathrm{L}$ of Reagent-1 (protein precipitation reagent for plasma; Jasem) was added and the mixture was again vortexed for $5 \mathrm{~s}$, then centrifuged at $4{ }^{\circ} \mathrm{C}$ (model NF1200R, rotor: RA 200, catalog No. B50 016; Nüve, Ankara, Turkey) at $10,000 \mathrm{rpm}(18,405 \times g)$ for $10 \mathrm{~min}$. After placing the resulting supernatant into a high-pressure liquid chromatography (HPLC) vial, $2 \mu \mathrm{L}$ was analyzed using a triple quadrupole Shimadzu LC-MS/MS 8045 instrument, which was operated with positive electronic spray ionization (+ESI) in multiple-reaction monitoring (MRM) mode implemented for the MS/MS detection of the analytes. The two mobile phases were acetonitrile/tetrahydrofuran $/ 25 \mathrm{mM}$ ammonium formate/formic acid (9/75/16/0.3) (phase A) and $100 \mathrm{mM}$ ammonium formate/acetonitrile (80/20) (phase B). A column with a spherical silica carrier ( $3 \mu \mathrm{m}$ particle size) of a unique stationary phase designed for amino acids was used for separation at a column temperature of $35{ }^{\circ} \mathrm{C}$ before MS identification (Intrada Amino Acid column; $3.0 \mathrm{~mm}$ internal 
diameter $\times 100 \mathrm{~mm}$; Imtakt), eluted at $0.6 \mathrm{~mL} / \mathrm{min}$ using the following program: $0 \% \mathrm{~B}(0-3.0 \mathrm{~min}), 0-17 \% \mathrm{~B}(3.0-9.0 \mathrm{~min})$, $17-100 \%$ B $(9.0-16.0 \mathrm{~min}), 100 \%$ B $(16.0-19.0 \mathrm{~min}), 0 \%$ B (19.50 $\mathrm{min})$. The standard concentrations were in the range values of $23-112 \mu \mathrm{mol} / \mathrm{L}$ for Asn, $5-150 \mu \mathrm{mol} / \mathrm{L}$ for Glu, $0-31 \mu \mathrm{mol} / \mathrm{L}$ for GABA, $127-341 \mu \mathrm{mol} / \mathrm{L}$ for Gly, $31-92 \mu \mathrm{mol} / \mathrm{L}$ for Phe, and 14-114 $\mu \mathrm{mol} / \mathrm{L}$ for Tyr. The ISTD contained all naturally occurring AAs except hydroxyproline (Hyp), hydroxylysine (OH-Lys), histidine (His), cystathionine (Cyst), taurine (Tau), and 1-methyl histidine (1-MIHS). Quantification of the analyte was conducted by means of calibration curves made on the concentrations of the calibrators with compensating for matrix effect and procedural losses according to yields of the assigned IS. Within the scope of the study, we measured the plasma levels of the following amino acids according to the following MRM $\mathrm{m} / \mathrm{z}$ criteria: Tau (126.2000>44.1000), tryptophan (Trp; 205.1000>188.0500), Phe (166.1000>120.0500), Tyr (182.1000>165.0000), leucine (Leu; 132.1000>43.1000), methionine (Met; 150.2000>104.1000), isoleucine (Ile; 132.1000>69.1000), Asn (133.2000>74.0500), proline (Pro; 116.1000>70.1000), glutamine (Gln; $148.2000>84.1000)$, GABA $(104.1000>87.1000)$, citrulline (Cit; 176.3000>159.1000), Glu (148.2000>84.1000), homocysteine (Hc2; 269.1000>136.0000), Cyst (223.1000>134.0000), cystine (Сy2; 241.2000>74.1000), arginine (Arg; $175.3000>70.1000)$, OH-Lys $(163.1000>128.1000)$, ornithine (Orn; 133.1000>70.1000), lysine (Lys; 147.1000>84.1000), His $\quad(156.1000>110.1000), \quad 3$-methylhistidine (3-MHIS; $170.1000>126.1000)$, 1 -MIHS $(170.1000>124.1000)$, valine (Val; 118.1000>72.1000), threonine (Thr; 120.1000>74.0500), serine (Ser; $106.2000>60.1000$ ), alanine (Ala; $90.1000>44.1000$ ), aspartic acid (Asp; $134.2000>74.2000)$, Gly (76.2000>30.1000), and Hyp $(132.2000>68.0000)$.

\section{Statistical analysis}

Statistical analyses were performed using IBM SPSS Statistics for Windows (version 24.0; IBM Corp). Descriptive statistics were summarized as number, percentage, mean, and standard deviation. The suitability of the variables to the normal distribution was investigated using visual (histogram and probability charts) and analytical methods (KolmogorovSmirnov). Normally distributed data were analyzed using a one-way analysis of variance (ANOVA), with post hoc Bonferroni correction. Analysis of variables without normal distribution was performed using a Kruskal-Wallis test. Categorical variables were analyzed using a Pearson chi-square or Fisher exact test (when any of the theoretical values observed on a $2 \times 2$ table was $<5$ ). Significance was accepted at a level of $P<0.05$ in all statistical analyses.

\section{Results}

We included 150 patients evaluated by the clinic for pediatric metabolic diseases and met the study criteria. Of the patients, $56 \%$ were boys and $44 \%$ were girls. At the time of admission to our clinic, the mean age of the patients was $6.55 \pm 4.35$ years (range 0-16). There were 44 (29\%) patients in the DC group and $56(37 \%)$ patients in the DIC group, and $50(34 \%)$ patients in the control group. When the groups were analyzed in terms of sex, weight for height percentile distribution, and mean age values, they were found to be similar (Table 1). In the DIC group, the rate of parental consanguinity was $87.5 \%$, while it was $75 \%$ in the DC group. The distribution of parental consanguinity rates between the 2 groups was found to be similar (Pearson chi-square test, $2 \times 2$ table, $P=0.106$ ). The rate of parental consanguinity for the control group was $22 \%$ and significantly lower than that of other groups (Pearson chisquare test, $3 \times 2$ table, $P<0.001$. The proportion of patients with siblings with a phenylketonuria history was $68 \%(n=38)$ in the DIC group, $20 \%(\mathrm{n}=9)$ in the DC group, and 4\% $(\mathrm{n}=2)$ in the control group. The proportion of patients with siblings with a phenylketonuria history was significantly higher in the DIC group than in the DC group (the Pearson chi-square test, $2 \times 2$ table, $P<0.001$ ). Only 8 patients had a history of an unexplained death of siblings during birth, all of whom were in the DIC group. When the patients were analyzed in terms of comorbidity and/or pathological condition, we found that 2 patients had epilepsy, 2 had mild mental motor retardation, and 1 had a hearing problem in the DIC group, while 1 patient had epilepsy and 2 had mild mental motor retardation in the DC group. None of the patients in the control group had any comorbidity or pathological condition.

In the analyses conducted, no significant difference was found between the DIC, DC, and control groups in terms of the mean Tau, Trp, Phe, Tyr, Leu, Met, Ile, Pro, Gln, Cit, Hc2, Cys, Cy2, Arg, OH-Lys, Orn, Lys, His, 3-MHIS, 1-MIHS, Val, Thr, Ser, Ala, Asp, or Hyp values (Table 2). When the mean Phe, Tyr, GABA, Gly, Asn, and Glu values of the DIC, DC, and control groups were compared, a significant difference was observed between the groups (Table 2). In post hoc analysis, a significant difference was found between the DIC, DC, and control groups in the mean Phe level (Table 3), but no significant difference was found between the DIC and DC groups in the mean Tyr level, while the mean Tyr level was significantly higher in the control group than both in the DIC and the DC groups (Table 3).

A significant difference was observed between the groups in the mean GABA, Gly, Glu and Asn levels (Table 2). In the post hoc analysis, the mean GABA and Gly values were significantly higher in the DIC group than they were in the DC 
Table 1. Sociodemographic characteristics of the patients

\begin{tabular}{|c|c|c|c|c|}
\hline & Diet incompatible group $(n=56)$ & Diet compatible group $(n=44)$ & Control group $(n=50)$ & $P$ \\
\hline Sex (male/female) & $30 / 26$ & $27 / 17$ & $27 / 23$ & $0.69+$ \\
\hline $\begin{array}{l}\text { Age }(\text { mean } \pm S D) \\
(\text { min-max) years }\end{array}$ & $6.57 \pm 3.99(0-14)$ & $6.75 \pm 4.62(0-16)$ & $6.36 \pm 4.58(0-16)$ & $0.15 \neq$ \\
\hline $\begin{array}{l}\text { Parental consanguinity } \\
\text { Yes/no n (\%) }\end{array}$ & $49(87.5) / 7(12.5)$ & $33(75) / 11(25)$ & $11(22) / 39(78)$ & $<0.001 \dagger$ \\
\hline Refugee status & & & & \\
\hline Yes/no n (\%) & $16(29) / 40(71)$ & $4(9) / 40(91)$ & $5(10) / 45(90)$ & $0.01 \dagger$ \\
\hline Lenght percentile ( $\mathrm{n}$ ) & & & & $0.96 \S$ \\
\hline $3-10 p$ & 8 & 5 & 5 & \\
\hline $10-25 p$ & 10 & 8 & 13 & \\
\hline $25-50 p$ & 14 & 14 & 16 & \\
\hline $50-75 p$ & 9 & 5 & 5 & \\
\hline $75-90 p$ & 6 & 6 & 6 & \\
\hline $90-97 p$ & 5 & 5 & 5 & \\
\hline Weight percentile (n) & & & & $0.82 \S$ \\
\hline $3-10 p$ & 6 & 5 & 5 & \\
\hline $10-25 p$ & 10 & 8 & 13 & \\
\hline $25-50 p$ & 12 & 14 & 17 & \\
\hline $50-75 p$ & 12 & 7 & 5 & \\
\hline $75-90 p$ & 9 & 5 & 5 & \\
\hline $90-97 p$ & 7 & 5 & 5 & \\
\hline
\end{tabular}

†Pearson chi-square test; łone-way ANOVA; §Fisher exact test; SD: standard deviation.

and control groups, and no significant difference was observed between the DC and control groups in the mean GABA and Gly levels (Table 3). In the post hoc analysis, the mean Glu and Asn levels were significantly higher in the DC and control groups than they were in the DIC group, while no significant difference was observed between the DC and control groups (Table 3).

The Pearson correlation analysis revealed a positive correlation between the GABA and Phe $(r=0.462, P<0.001)$ and Gly and Phe $(r=0.511, P<0.001)$ levels (Table 4). Moreover, a negative correlation was found between the Glu and Phe $(r=-0.475, p<0.001)$ and Asp and Phe $(r=-0.412$, $P<0.001)$ levels in the Pearson correlation analysis (Table 4).

\section{Discussion}

Numerous congenital metabolic diseases can be diagnosed by using a dried blood spot (heel prick) test in the early neonatal period. Early diagnosis of phenylketonuria is extremely important, especially as it is an important cause of preventable mental retardation. Phenylketonuria is common in geographical regions where consanguineous marriages, such as in Turkey and Iran, are frequent $[16,17]$. We found that the rates of consanguineous marriages in the parents and a history of phenylketonuria in siblings were higher in children with classic phenylketonuria than they were in healthy children.

Compliance with dietary treatment is most effective in infancy and childhood [18]. The diet severely interferes with culturally normal eating habits, particularly in older children and adolescents, and this often results in problems with compliance with dietary treatment recommendations [5, 18]. In the present study, the ages of the patients in the DC and DIC groups were similar. We consider that sociocultural factors, such as seasonal agricultural work and refugee life, may affect this situation. Indeed, in the present study, we found that refugees were more common among the DIC group patients.

Especially in regions where phenylketonuria is more common, besides early diagnosis, close follow-up of patients is also necessary. Depending on the age of the patients and their adherence to the diet (blood Phe level), white matter abnormalities (WMA) may arise in the brain tissue of patients with phenylketonuria, and cognitive functions of children may be adversely affected [19-21]. Although the pathogenesis of resulting WMA and the severity of adversely affected cognitive functions have not been fully elucidated, it has been shown that WMA may regress within 6 months with decreased blood Phe levels [3, 19, 22]. Various scoring tests and cranial magnetic resonance imaging (MRI) techniques are used in the follow-up of a patient's cognitive functions [2]. 
Table 2. Mean serum levels of amino acids in phenyketonuric patients and controls

\begin{tabular}{|c|c|c|c|c|}
\hline Plasma amino acid ( $\mu \mathrm{mol} / \mathrm{L})$ & Diet incompatible group $(n=56)$ & Diet compatible group $(n=44)$ & Control group $(n=50)$ & $P$ \\
\hline Taurine & $72.46 \pm 26.49$ & $73.54 \pm 30.14$ & $79.69 \pm 24.37$ & 0.34 \\
\hline Tryptophan & $52.11 \pm 23.1$ & $53.67 \pm 19.63$ & $48.64 \pm 14.29$ & 0.43 \\
\hline Phenylalanine & $845.87 \pm 380.03$ & $162.68 \pm 105.51$ & $57.67 \pm 16.76$ & $<0.001^{* * *}$ \\
\hline Tyrosine & $53.7 \pm 40.12$ & $54.33 \pm 24.72$ & $69.09 \pm 26.96$ & $<0.001^{* * *}$ \\
\hline Leucine & $106.04 \pm 55.11$ & $94.27 \pm 34.82$ & $104.43 \pm 33.26$ & 0.35 \\
\hline Methionine & $25.26 \pm 10.69$ & $25.66 \pm 7.68$ & $28.13 \pm 9.87$ & 0.26 \\
\hline Isoleucine & $49.97 \pm 24.4$ & $41.98 \pm 14.92$ & $46.38 \pm 17.95$ & 0.14 \\
\hline Asparagine & $39.3 \pm 16.89$ & $56.33 \pm 21.3$ & $59.79 \pm 20.64$ & $<0.001^{* * *}$ \\
\hline Proline & $213.87 \pm 128.52$ & $242.80 \pm 103.59$ & $246.58 \pm 126.68$ & 0.31 \\
\hline Glutamine & $481.21 \pm 126.66$ & $511.62 \pm 124.11$ & $467.61 \pm 120.82$ & 0.22 \\
\hline GABA & $0.96 \pm 1.07$ & $0.39 \pm 0.7$ & $0.32 \pm 0.57$ & $<0.001^{* * *}$ \\
\hline Citrulline & $32.93 \pm 10.9$ & $29.35 \pm 8.7$ & $31.99 \pm 14.95$ & 0.31 \\
\hline Glutamic acid & $39.01 \pm 22.94$ & $80.47 \pm 31.92$ & $98.48 \pm 42.54$ & $<0.001^{* * *}$ \\
\hline Homocystin & $0.14(0-1.85)$ & $0.23(0-0.99)$ & $0.18(0-1.96)$ & 0.2 \\
\hline Cystathionine & $0.24(0-0.85)$ & $0.3(0-1.02)$ & $0.25(0-1.36)$ & 0.67 \\
\hline Cystine & $30.46 \pm 13.13$ & $31.77 \pm 11.84$ & $30.08 \pm 9.86$ & 0.75 \\
\hline Arginine & $68.69 \pm 25.75$ & $72.84 \pm 30.41$ & $77.69 \pm 27.13$ & 0.25 \\
\hline Hydroxylysine & $1.23(0-32.58)$ & $1.18(0-19.97)$ & $1.25(0-11.3)$ & 0.13 \\
\hline Ornithine & $69.99 \pm 22.92$ & $72.87 \pm 31.58$ & $79.32 \pm 26.54$ & 0.13 \\
\hline Lysine & $138.69 \pm 63.3$ & $127.94 \pm 50.06$ & $121.52 \pm 37.65$ & 0.65 \\
\hline Histidine & $58.14 \pm 34.44$ & $53.05 \pm 21.52$ & $50.82 \pm 25.91$ & 0.17 \\
\hline 3-methylhistidine & $1.86(0.36-16.8)$ & $1.71(0.16-4.39)$ & $1.98(0.30-5.95)$ & 0.34 \\
\hline 1-methylhistidine & $1.35(0.23-5.99)$ & $1.1(0.48-7.47)$ & $1.04(0.42-9.74)$ & 0.72 \\
\hline Valine & $190.56 \pm 91.52$ & $169.54 \pm 62.43$ & $171 \pm 55.76$ & 0.26 \\
\hline Threonine & $109.75 \pm 56.74$ & $117.71 \pm 38.93$ & $114.67 \pm 45.54$ & 0.70 \\
\hline Serine & $132.62 \pm 40.3$ & $140.07 \pm 42.70$ & $144.5 \pm 31.99$ & 0.30 \\
\hline Alanine & $321.10 \pm 129.26$ & $372.5 \pm 136.02$ & $345.72 \pm 145.27$ & 0.17 \\
\hline Aspartic acid & $11.91 \pm 7.19$ & $12.38 \pm 6.98$ & $13.1 \pm 10.26$ & 0.75 \\
\hline Glycine & $305.1 \pm 105.19$ & $202.82 \pm 73.99$ & $174.73 \pm 102.28$ & $<0.001^{* * *}$ \\
\hline Hydroxyproline & $9.50 \pm 3.35$ & $9.94 \pm 3.95$ & $9.98 \pm 2.35$ & 0.7 \\
\hline
\end{tabular}

SD, Standard deviation; GABA, $\gamma$-aminobutyric acid.

Mean \pm SD and $P$-value for the one-way ANOVA and median (min-max) and its $P$-value for the Kruskal-Wallis test are given.

***P $P<0.001$.

While the blood Phe level can be measured precisely from venous blood using LC-MS/MS, many other variables can be measured simultaneously. One of these variables is the blood Tyr level. Current recommendations are to add Tyr to the diet in the treatment of patients with phenylketonuria [2]. In our study, the mean plasma Tyr levels in the patients with phenylketonuria (DIC and DC groups) were found to be lower than that of participants in the control group, suggesting that Tyr supplement added to the diet might be clinically beneficial in patients with phenylketonuria. In the present study, the plasma levels of neurotransmitter AAs, which are considered to play an important role in the CNS functions, have been investigated along with the blood Phe level. Yuan et al. [23] revealed that decreased GABA level and increased Asp, Glu, Tau, and L-Ser levels in plasma could be correlated with Parkinson's disease. Bugajska et al. [24] found a correlation between low plasma exogenous AA levels and autism in a pediatric population, so that there may be a correlation between autism and phenylketonuria [25]. Zaki et al. [26] found that the levels of some AAs in autistic children, other than Phe in plasma, differed from those in healthy children. A study demonstrating a correlation between major depressive disorder (MDD) and 
Table 3. Post hoc analysis of blood plasma amino acid levels with significant differences

\begin{tabular}{lccc}
\hline Amino acid & DIC-DC & DIC-Control & DC-Control \\
\hline Phenylalanine & $<0.001$ & $<0.001$ & $<0.001$ \\
Tyrosine & $>0.99$ & $<0.001$ & 0.004 \\
GABA & 0.005 & $<0.001$ & 0.92 \\
Glycine & $<0.001$ & $<0.001$ & 0.47 \\
Asparagine & $<0.001$ & $<0.001$ & 0.81 \\
Glutamic acid & $<0.001$ & $<0.001$ & 0.06 \\
\hline
\end{tabular}

DC, diet compatible group; DIC, diet incompatible group; GABA, $\gamma$-aminobutyric acid.

One-way ANOVA with Bonferroni post hoc correction applied.

Table 4. Correlation between plasma levels of phenylalanine and other amino acids

\begin{tabular}{lcc}
\hline Correlated factors & $\boldsymbol{r}$ & $\boldsymbol{P}$ \\
\hline Phenylalanine-GABA & 0.462 & $<0.001$ \\
Phenylalanine-glycine & 0.511 & $<0.001$ \\
Phenylalanine-asparagine & -0.412 & $<0.001$ \\
Phenylalanine-glutamic acid & -0.475 & $<0.001$ \\
\hline
\end{tabular}

$r$, Pearson correlation coefficient; GABA: $\gamma$-aminobutyric acid.

plasma AA levels was conducted by Ogawa et al. [27]. They found that plasma Asn levels were lower, whereas Glu levels were higher in children with MDD than they were in healthy children.

As is seen in various studies, plasma AA levels may vary in many diseases affecting the CNS. To our knowledge, the present study is the first to show the relationship between dietary compliance and plasma excitatory and inhibitory AA levels in patients with phenylketonuria. We found that the mean plasma GABA and Gly values were higher, and the mean plasma Glu and Asn values were lower in the DIC group than they were in the DC and control groups. As expected, no significant difference in levels between the DC and control groups was observed, except for the mean plasma levels of Phe and Tyr. A positive correlation was found between plasma Phe level and both GABA and Gly levels, whereas we found a negative correlation between plasma Phe levels and both Glu and Asn levels.

Our present study is limited because no intelligence test was performed on the patients in this situation, so any correlation between plasma Phe level and intelligence test score could not be evaluated. Failure to determine cerebrospinal fluid levels of neurotransmitters and lack of results of neuromotor development tests (e.g., Denver) of patients can be considered as a limitation. Socioeconomic parameters, such as parental education level and monthly income, which might affect dietary compliance, were not evaluated in the present study. Multicenter studies to be conducted with plasma AA analysis results of patients with phenylketonuria in different clinical centers are warranted.

\section{Conclusions}

High blood plasma levels of inhibitory AAs, such as GABA and Gly, and low plasma levels of excitatory AAs, such as Asn and Glu, were measured in patients in Sanliurfa province, Turkey, with phenylketonuria who did not follow their diets. The plasma levels of excitatory and inhibitory AAs may be used as auxiliary parameters in the follow-up of cognitive functions of patients with phenylketonuria. More extensive studies are needed to demonstrate any negative association of cognitive function and the plasma levels of these AAs.

Author contributions. $\mathrm{HK}$ and $\mathrm{MK}$ conceived and designed the study, collected, analyzed, and interpreted the data. Both authors contributed to drafting and critical revision of the manuscript, approved the final version submitted for publication, and take responsibility for the statements made in the published article.

Acknowledgments. We thank Dr. Ataman Gonel, president of the Department of Biochemistry, Harran University Medical School, and the staff of the biochemistry laboratory for their advice and help with the analyses. We did not receive any specific grant for this research from any funding agencies in the public, commercial, or not-for-profit sectors.

Conflict of interest statement. The authors have each completed and submitted an International Committee of Medical Journal Editors Uniform Disclosure Form for Potential Conflicts of Interest. Neither of the authors discloses any potential or actual conflict of interest. No financial or nonfinancial benefits have been or will be received from any party related directly or indirectly to the subject of this article.

Data sharing statement. The datasets generated or analyzed during the current study are available from the corresponding author on reasonable request.

\section{References}

[1] Blau N, van Spronsen FJ, Levy HL. Phenylketonuria. Lancet. 2010; 376(9750):1417-27. 
[2] van Wegberg AMJ, MacDonald A, Ahring K, Bélanger-Quintana A, Blau N, Bosch AM, et al. The complete European guidelines on phenylketonuria: diagnosis and treatment. Orphanet J Rare Dis. 2017; 12:162. doi: 10.1186/s13023-017-0685-2

[3] van Vliet D, van Wegberg AMJ, Ahring K, Bik-Multanowski M, Blau N, Bulut FD, et al. Can untreated PKU patients escape from intellectual disability? A systematic review. Orphanet J Rare Dis. 2018; 13:149. doi: 10.1186/s13023-018-0890-7

[4] Didycz B, Bik-Multanowski M. Dynamics of hyperphenylalaninemia and intellectual outcome in teenagers with phenylketonuria. Acta Biochim Pol. 2017; 64:527-31.

[5] Burgard P, Lachmann RH, Walter J. Hyperphenylalaninaemia. In: Saudubray JM, Van den Berghe G, Walter J, editors. Inborn metabolic diseases: diagnosis and treatment. 6th ed. New York: Springer; 2016, p. 253-4.

[6] MacDonald A, Singh RH, Rocha JC, van Spronsen FJ. Optimising amino acid absorption: essential to improve nitrogen balance and metabolic control in phenylketonuria. Nutr Res Rev. 2019; 32:70-8.

[7] de la Parra A, García MI, Hamilton V, Arias C, Cabello JF, Cornejo V. First-year metabolic control guidelines and their impact on future metabolic control and neurocognitive functioning in children with PKU. Mol Gent Metab Rep. 2017; 13:90-4.

[8] Pinto A, Adams S, Ahring K, Allen H, Almeida MF, Garcia-Arenas $\mathrm{D}$, et al. Early feeding practices in infants with phenylketonuria across Europe. Mol Genet Metab Rep. 2018; 16:82-9.

[9] Uaariyapanichkul J, Chomtho S, Suphapeetiporn K, Shotelersuk V, Punnahitananda S, Chinjarernpan P, Suteerojntrakool O. Age-related reference intervals for blood amino acids in Thai pediatric population measured by liquid chromatography tandem mass spectrometry. J Nutr Metab. 2018; 2018:5124035. doi: $10.1155 / 2018 / 5124035$

[10] Fingerhut R, Silva Polanco ML, Silva Arevalo Gde J, Swiderska MA. First experience with a fully automated extraction system for simultaneous on-line direct tandem mass spectrometric analysis of amino acids and (acyl-)carnitines in a newborn screening setting. Rapid Commun Mass Spectrom. 2014; 28:965-73.

[11] Zabielski P, Ford GC, Persson XM, Jaleel A, Dewey JD, Nair KS. Comparison of different mass spectrometry techniques in the measurement of L-[ring- ${ }^{13} \mathrm{C}_{6}$ ] phenylalanine incorporation into mixed muscle proteins. J Mass Spectrom. 2013; 48:269-75.

[12] Eggers AE. A serotonin hypothesis of schizophrenia. Med Hypotheses. 2013; 80:791-4.

[13] Cao B, Wang D, Brietzke E, McIntyre RS, Pan Z, Cha D, et al. Characterizing amino-acid biosignatures amongst individuals with schizophrenia: a case-control study. Amino Acids. 2018; 50:1013-23.

[14] Belanger AM, Przybylska M, Gefteas E, Furgerson M, Geller S, Kloss A, et al. Inhibiting neutral amino acid transport for the treatment of phenylketonuria. JCI Insight. 2018; 3:e121762. doi: $10.1172 /$ jci.insight. 121762
[15] González García MB, Conde-Guzon P, Alcalde Martín C, Conde-Guzon MJ, Velasco Zúñiga R. Neuropsychological assessment among children and adolescents with phenylketonuria and hyperphenylalaninemia and its relationship with plasma phenylalanine levels. Arch Argent Pediatr. 2017; 115:267-73.

[16] Keyfi F, Nasseri M, Nayerabadi S, Alaei A, Mokhtariye A, Varasteh A. Frequency of inborn errors of metabolism in a Northeastern Iranian sample with high consanguinity rates. Hum Hered. 2018; 83:71-8.

[17] Tezel B, Dilli D, Bolat H, Sahman H, Ozbaş S, Acıcan D, et al. The development and organization of newborn screening programs in Turkey. J Clin Lab Anal. 2014; 28:63-9.

[18] Walkowiak D, Bukowska-Posadzy A, Kałużny Ł, Ołtarzewski M, Staszewski R, Musielak M, et al. Therapy compliance in children with phenylketonuria younger than 5 years: a cohort study. Adv Clin Exp Med. 2019; 28:1385-91.

[19] Peng H, Peck D, White DA, Christ SE. Tract-based evaluation of white matter damage in individuals with early-treated phenylketonuria. J Inherit Metab Dis. 2014; 37:237-43.

[20] Sadek AA, Hassan MH, Mohammed NA. Clinical and neuropsychological outcomes for children with phenylketonuria in Upper Egypt; a single-center study over 5 years. Neuropsychiatr Dis Treat. 2018; 14:2551-61.

[21] Romani C, Palermo L, MacDonald A, Limback E, Hall SK, Geberhiwot T. The impact of phenylalanine levels on cognitive outcomes in adults with phenylketonuria: Effects across tasks and developmental stages. Neuropsychology. 2017; 31:242-54.

[22] White DA, Antenor-Dorsey JA, Grange DK, Hershey T, Rutlin J, Shimony JS, et al. White matter integrity and executive abilities following treatment with tetrahydrobiopterin (BH4) in individuals with phenylketonuria. Mol Genet Metab. 2013; 110:213-7.

[23] Yuan YS, Zhou XJ, Tong Q, Zhang L, Zhang L, Qi ZQ, et al. Change in plasma levels of amino acid neurotransmitters and its correlation with clinical heterogeneity in early Parkinson's disease patients. CNS Neurosci Ther. 2013; 19:889-96.

[24] Bugajska J, Berska J, Wojtyto T, Bik-Multanowski M, Sztefko K. The amino acid profile in blood plasma of young boys with autism. Psychiatr Pol. 2017; 51:359-68.

[25] Khemir S, Halayem S, Azzouz H, Siala H, Ferchichi M, Guedria A, et al. Autism in phenylketonuria patients: from clinical presentation to molecular defects. J Child Neurol. 2016; 31:843-9.

[26] Zaki MM, Abdel-Al H, Al-Sawi M. Assessment of plasma amino acid profile in autism using cation-exchange chromatography with postcolumn derivatization by ninhydrin. Turk J Med Sci. 2017; 47:260-7.

[27] Ogawa S, Koga N, Hattori K, Matsuo J, Ota M, Hori H, et al. Plasma amino acid profile in major depressive disorder: analyses in two independent case-control sample sets. J Psychiatr Res. 2018; 96:23-32. 